\title{
Development of a luciferase/luciferin cell proliferation (XenoLuc) assay for real-time measurements of Gfp-Luc2-modified cells in a co-culture system
}

Sin-Yeang Teow ${ }^{1,2}$, Kitson Liew ${ }^{1}$, Mohd Firdaus Che Mat ${ }^{1}$, Marini Marzuki ${ }^{1}$, Norazlin Abdul Aziz ${ }^{1}$, Tai-Lin Chu', Munirah Ahmad ${ }^{1}$ and Alan Soo-Beng Khoo ${ }^{1 *}$ (i)

\begin{abstract}
Background: In vitro modelling of cancer cells is becoming more complex due to prevailing evidence of intimate interactions between cancer cells and their surrounding stroma. A co-culture system which consists of more than one cell type is physiologically more relevant and thus, could serve as a useful model for various biological studies. An assay that specifically detects the phenotypic changes of cancer cells in a multi-cellular system is lacking for nasopharyngeal carcinoma (NPC).

Results: Here, we describe a luciferase/luciferin (XenoLuc) assay that could specifically measure changes in the proliferation of cancer cells in the co-culture system using two modified NPC patient-derived tumour xenograft (PDTXs) cells: Xeno284-gfp-luc2 and XenoB110-gfp-luc2. Through this assay, we are able to show that the growth of NPC xenograft cells in both two-dimensional (2D) and three-dimensional (3D) models was enhanced when cocultured with normal human dermal fibroblasts (NHDFs). In addition, potential applications of this assay in in vitro drug or inhibitor screening experiments are also illustrated.

Conclusions: XenoLuc assay is specific, sensitive, rapid and cost-effective for measuring the growth of luciferaseexpressing cells in a co- or multiple-culture system. This assay may also be adapted for tumour microenvironment studies as well as drug screening experiments in more complex 3D co-culture systems.
\end{abstract}

Keywords: Luciferase assay, Luminescence, Nasopharyngeal carcinoma, Nasopharyngeal neoplasms, Patient-derived xenograft, Fibroblasts, Tumour microenvironment, Stromal interaction, Proliferation, Co-culture, Spheroids, Drug screening, Cancer immunology

\section{Background}

Cell-based assays remain the leading platform for cancer research in preclinical settings. Majority of highthroughput compound screening programmes carried out by major pharmaceutical companies utilise cellbased assays to identify high-quality leads [1]. Such assays have the advantage over biochemical-based assays since these assays provide information on the cellular

\footnotetext{
*Correspondence: alankhoo@imr.gov.my; alankhoo.imr@gmail.com ${ }^{1}$ Molecular Pathology Unit, Cancer Research Centre, Institute for Medical Research, National Institutes of Health (NIH Complex), Ministry of Health Malaysia, Level 4, Block C7, No: 1, Jalan Setia Murni U13/52, Section U13, Setia Alam, 40170 Shah Alam, 50588 Kuala Lumpur, Selangor, Malaysia Full list of author information is available at the end of the article
}

responses of a particular drug candidate. Moreover, cellbased assays could be reliably used to provide early indications of drug toxicity [1]. The recent advancement in cell culture technology has paved way for the development of in vitro cell-based assays that could be carried out in a more biologically relevant microenvironments. Such assays may include the incorporation of extracellular matrices (ECMs) and scaffolds as well as stromal cells in the presence of tumour cells [2, 3]. However, challenges remain in the design of these assays, particularly on measuring phenotypic changes of a specific cell population in complex multi-cell type systems. Notably, there is a scarcity of convenient cell-based assays that

(C) The Author(s). 2019 Open Access This article is distributed under the terms of the Creative Commons Attribution 4.0 International License (http://creativecommons.org/licenses/by/4.0/), which permits unrestricted use, distribution, and 
could discriminate phenotypes of different cell types in a co-culture setting $[4,5]$. With greater understanding of the microenvironment surrounding a tumour, it is increasingly important to employ appropriate in vitro assays that are able to address issues of tumour complexity and heterogeneity [2].

Cancer cells are often grown as monolayers, of which 2D (monolayer) culture models are commonly employed for in vitro drug testing. However, these 2D models are bound by several limitations that may potentially negate their usefulness. For example, there is a lack of 3D cellcell and cell-ECM interactions in 2D models that are present in in vivo models [6]. These interactions generate signalling cues that are pivotal for numerous important cellular functions such as proliferation, differentiation and survival. Most of these functions are lost or have been compromised in 2D culture models. As a result, data generated from $2 \mathrm{D}$ in vitro drug testing could be misleading and non-predictive for in vivo responses [6]. The spatial arrangement of cancer cells within the 3D model, possibly together with the presence of other cell types and ECM components, mimic their natural microenvironment and hence, may reinstate the important signalling that are lost or compromised in 2D culture systems [7]. For these reasons, 3D culture models offer greater clinical and biological relevance than in vitro models and therefore, could bridge the gap between the monolayer culture and animal works.

Our group had developed two PDTXs, XenoB110 and Xeno284 from Malaysian NPC patients [8]. To facilitate the monitoring of in vivo tumour growth, these PDTXs were co-transduced with gfp-luc2 reporter genes (XenoB110-gfp-luc2 and Xeno284-gfp-luc2). The XenoLuc assay described in this paper is a luciferase-based assay that uses commercial D-Luciferin as the substrate for the luciferase in the transduced PDTXs to generate a luminescent signal that is proportional to cell numbers. We demonstrate that the XenoLuc assay is sensitive and could specifically measure real-time proliferation of PDTXs both in vitro and in vivo. As 3D models are gaining prominence as in vitro cell-based assays, we also demonstrate the robustness of XenoLuc assay in both mono- and multi-cellular spheroid cultures in addition to the conventional 2D monolayer model. This assay is able to measure the PDTXs growth enhancement that resulted from the addition of growth supplements as well as from the effect of co-culturing with other human cell types. In addition, the assay may also be used to evaluate the loss of cell viability, such as that induced by cisplatin treatment. Since the proliferation and viability of PDTX cells could be specifically detected in the complex 3D co-culture systems, XenoLuc assay may represent a cost-effective and a potentially useful cell-based assay for studies on tumour microenvironment, high- throughput compound screening and preclinical drug response prediction.

\section{Results \\ Specificity and sensitivity of XenoLuc assay}

The specificity of XenoLuc assay was assessed by determining if it specifically detected luminescent signals from luciferase-expressing cells compared to nonexpressing cells. All experiments were carried out using freshly isolated xenograft cells harvested from three separate tumours from different mice and used for not more than two passages. Non-treated/non-cultured cells are used in assays as internal controls to avoid batch-tobatch variation which could arise from multiple causes. Based on Fig. 1a, luminescence was detected in luc2transduced xenograft cells but not in parental xenograft cells and NHDFs, indicating the specificity of XenoLuc assay. Additional file 1: Figure S1 shows the linear correlation between luminescence signals versus various titrations of XenoB110-gfp-luc2 and Xeno284-gfp-luc2 cells measured at day- 0 (30 min after cell seeding when the cells had settled at the bottom of wells, but had not adhered). The mouse cell-depleted xenograft cells exhibited higher luminescent signals compared to the nondepleted xenograft cells (Fig. 1a and b). To examine the assay sensitivity, two-fold serial dilutions of cells were plated and the readings were taken after 4 days. Figure $1 \mathrm{~b}$ shows that cell seeding number had a positive linear correlation with luminescence in both $2 \mathrm{D}$ and $3 \mathrm{D}$ culture models, of which the lowest seeding density tested was 2500 cells/well in a 96-well plate. Overall in 2D and 3D models, XenoB110-gfp-luc2 cells exhibited higher signals (about 20-fold) than Xeno284-gfp-luc2 cells. This could be due to a higher expression of luciferase in XenoB110gfp-luc2 cells.

We then asked whether XenoLuc assay could be used to measure cell proliferation. Figure 1c shows that the luminescent signals increased in a time-dependent manner whereby the maximum growth of both xenograft cells was recorded at Day 6 and Day 4 for 2D and 3D culture models, respectively. Following that, the growth of both xenograft cells either reached plateau phase or declined. In another experiment, we captured the images of XenoB110-gfp-luc2 cells (2D and 3D cultures) under FITC channel using IN-Cell high content cellular analyser, and the GFP images were subjected to fluorescence intensity analysis by the IN-Cell Developer software. Additional file 2: Figure S2A and S2B show that the fluorescence intensity of non-depleted and mouse celldepleted xenograft cells in 2D culture correlated positively to the number of cells seeded. The signal intensity also increased following the increase in cell proliferation from day 2 to 4 . Similar correlations (between signal intensity and cell seeding numbers) were seen when 


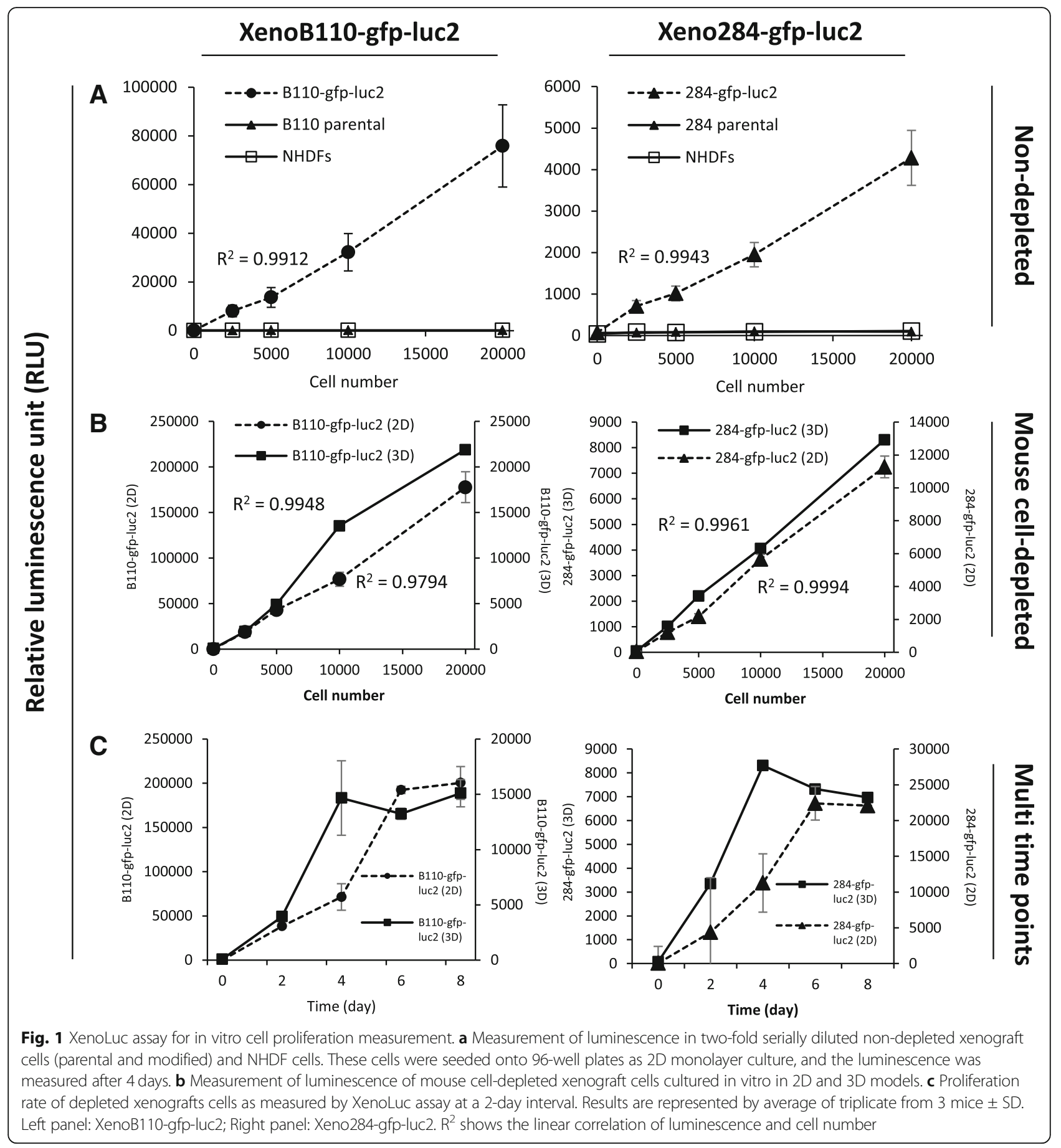

XenoB110-gfp-luc2 cells were grown as 3D spheroids (Additional file 2: Figure S2C). However, in these 3D cultures, unlike luminescence signals, no obvious difference was found in fluorescence intensity between day- 2 and day- 4 readings.

\section{Characterization of XenoLuc assay}

The principle of XenoLuc assay was illustrated in Fig. 2a. Light or luminescence is generated as the result of the enzymatic reaction between the D-Luciferin substrate and the intracellular luciferase in the presence of oxygen and adenosine triphosphate (ATP). In addition to the luminescence, other by-products are oxyluciferin, AMP, PPi (pyrophosphate), and carbon dioxide [9]. Figure 2b shows that the XenoLuc luminescent assay may be used either as endpoint (lytic) or non-destructive (non-lytic) assays. In line with the current trend of multiplexing cell-based assays, the non-lytic assay is preferable to the 

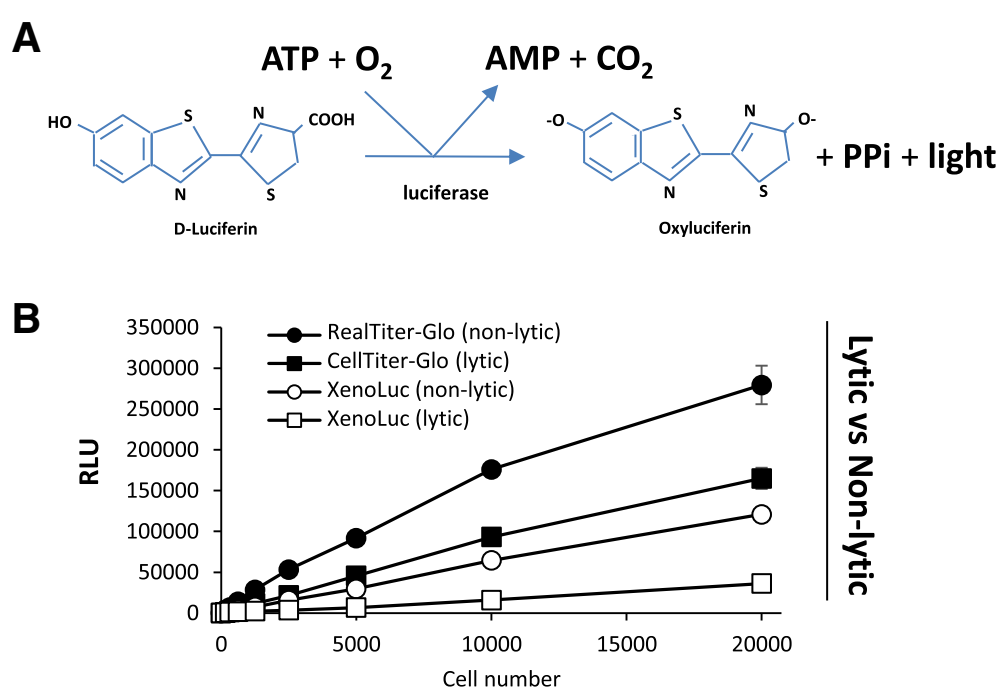

C
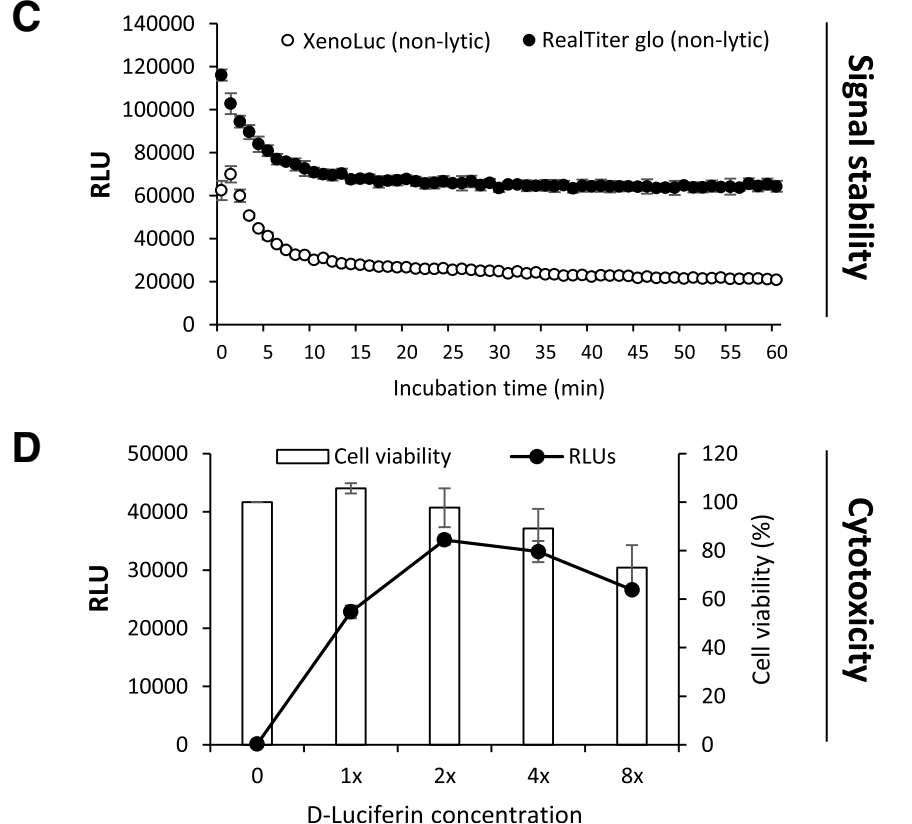

Fig. 2 Characterization of XenoLuc assay. a Diagram showing the basic concept of luciferase/luciferin system. b Comparison of signal intensity detected by XenoLuc assay and two commercial assays: CellTiter-Glo (lytic) and RealTime-Glo (non-lytic). c Comparison of signal stability of XenoLuc and RealTime-Glo. d Cytotoxicity of D-Luciferin substrate after 24-h incubation as determined by MTS assay. Results are represented by average of triplicate from 3 mice \pm SD

traditional endpoint assay because no cell lysis is required, and the same well may be multiplexed for various assays [10]. Comparatively, the luminescent signals detected by XenoLuc assay were relatively lower than that of commercial assays (Fig. 2b). RealTime-Glo and CellTiter-Glo employ exogenous luciferase and possibly more sensitive proprietary luciferin substrate along with other signal enhancers or co-factors such as $\mathrm{Mg}^{2+}$, recombinant ATP and Coenzyme A (CoA) [11]. The nonlytic version of XenoLuc assay was found to give higher signals than the lytic version of the assay at various tested cell numbers (Fig. 2b). We then assessed the luminescent signal stability of the XenoLuc assay in comparison with RealTime-Glo by recording the signals for $60 \mathrm{~min}$ at a 1-min interval. Figure $2 \mathrm{c}$ shows that the signals in both assays initially dropped from the first reading, and eventually stabilized after $10 \mathrm{~min}$ of incubation. These signals were stable for up to $60 \mathrm{~min}$. We then selected $10 \mathrm{~min}$ as the incubation time for luciferin substrate with the luc2-expressing cells for subsequent experiments. As shown in Fig. 2d, the increase of Dluciferin substrate concentration to $2 \mathrm{X}$ from $1 \mathrm{X}$ increased the luminescence signal by 1.5 folds. The increment in signal declined when D-luciferin substrate 
was used at higher concentrations of $4 \mathrm{X}$ and $8 \mathrm{X}$. More importantly, the presence of luciferin at $2 \mathrm{X}$ for $24 \mathrm{~h}$ did not cause toxicity to XenoB110-gfp-luc2 cells (Fig. 2d). This is consistent with Tiffen et.al [12]. However, cell viability was reduced to 89 and $73 \%$, respectively when incubated with $4 \mathrm{X}$ and $8 \mathrm{X}$ luciferin substrate (Fig. $2 \mathrm{~d}$ ). This explains the reduction in luminescent signals at these concentrations. Similar trends were also observed in Xeno284-gfp-luc2 cells (data not shown).

\section{XenoLuc assay for anti-cancer drug screening}

Figure 3 shows that cisplatin exhibited dose-dependent cytotoxicity against both xenograft cells. Of the two xenograft cells, the decrease in cell viability of Xeno284gfp-luc2 cells were less possibly because the cells were more resistant to cisplatin treatment $\left(\mathrm{IC}_{50}\right.$ of $45 \mu \mathrm{M}$ and $30 \mu \mathrm{M})$ as compared to XenoB110-gfp-luc2 cells ( $\mathrm{IC}_{50}$ of $2.5 \mu \mathrm{M}$ and $6.25 \mu \mathrm{M})$ in $2 \mathrm{D}$ and $3 \mathrm{D}$ culture models. A parallel comparison revealed that the overall luminescence of Xeno284-gfp-luc2 cells was lower than XenoB110-gfp-luc2 cells, consistent with Fig. 1. Nevertheless, comparisons were made possible because of the inclusion of untreated cells derived from the same cell isolates and carried out within the same experimental batch to avoid batch-to-batch variability.

\section{Determination of xenograft cell growth in a co-culture system}

As demonstrated by Fig. 2, XenoLuc assay specifically detected luc2-bearing tumour cells. Additionally, it

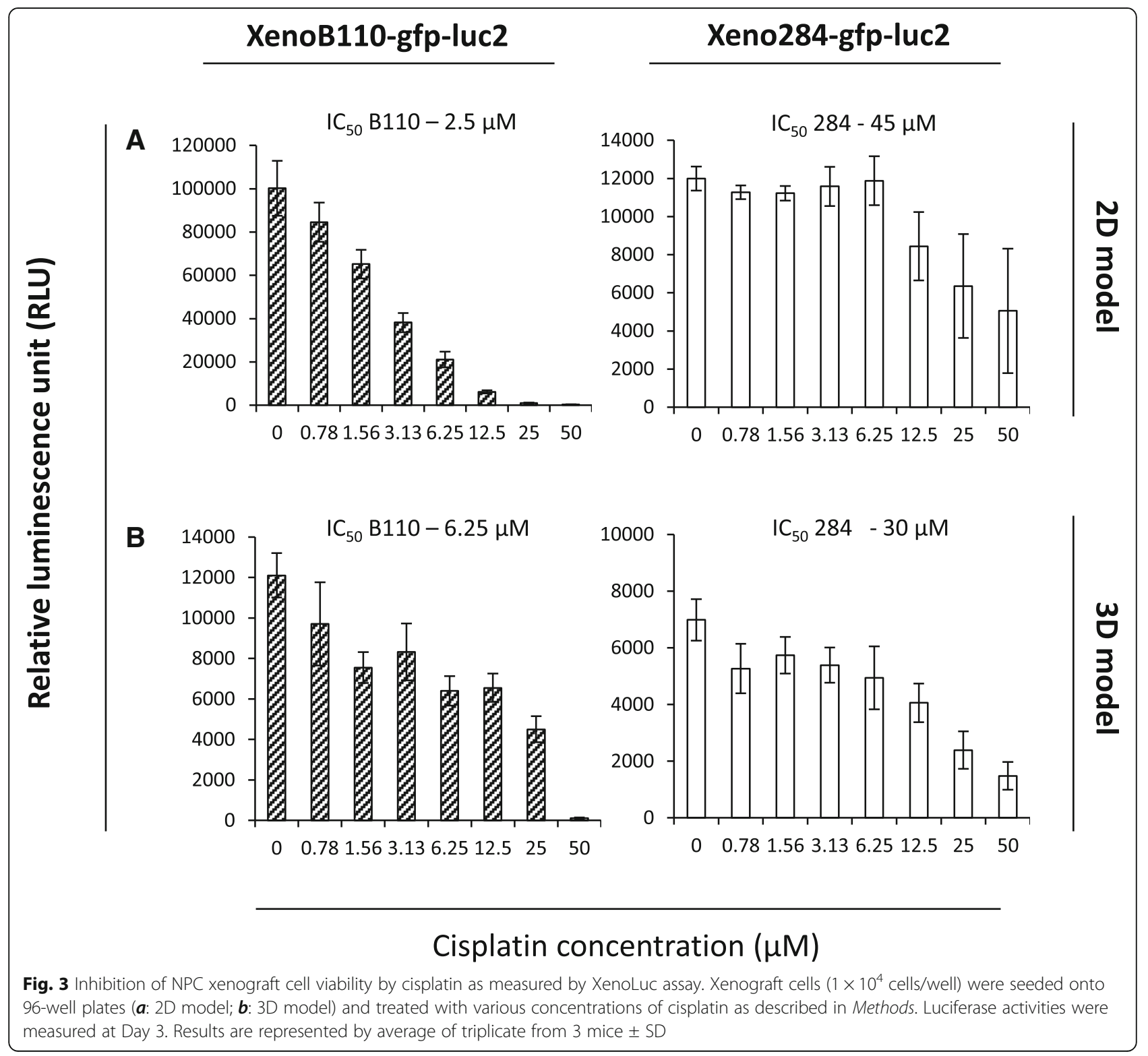


would be beneficial if it could be used to measure the growth of tumour cells (either inhibition or enhancement) that were co-cultured with other cell types. To validate the use of XenoLuc assay in this application, we co-cultured the xenograft cells with either fibroblasts (NHDFs) (adherent cells) or PBMCs (suspension cells), and measured the luminescence signals to examine the growth inhibition or enhancement of xenograft cells. Figure 4a shows that the NHDFs enhanced xenograft cell growth in 2D culture model. However, only XenoB110-gfp-luc2 cells displayed a significant growth enhancement $(p<0.05)$. The growth enhancement in XenoB110-gfp-luc2 cells was also dependent on the number of seeded NHDFs (Additional file 3: Figure S3A). Coculture of xenograft cells with PBMCs did not yield any significant difference in the growth as compared to that of monoculture (Fig. 4b and Additional file 3: Figure S3B). We then investigated whether this enhancing effect was reproducible in 3D culture model. Similarly, NHDFs significantly promoted XenoB110-gfp-luc2 cell growth in a seeding number-dependent manner $(p<0.05)$ (Fig. $4 \mathrm{~b}$ and Additional file 3: Figure S3B). Figure 5 shows the microscopic analysis of GFP-labelled xenograft cells cocultured with NHDFs and PBMCs individually in 3D models. Xenograft cell-NHDF co-cultures resulted in irregular and multiple spheroids compared to xenograft cell-PBMC co-cultures. Consistent with the luminescence data, the fluorescence intensity analysis showed that NHDFs promoted the growth of XenoB110-gfp-luc2 cells in both 2D and 3D cultures (Additional file 4: Figure S4).

\section{Discussion}

In the present study, we developed a specific and sensitive luciferase-based assay known as XenoLuc to detect and measure real-time proliferation of luc2-transduced cells. The assay used freshly isolated xenograft cells which were passaged for not more than two times. This approach was established to circumvent the difficulty of

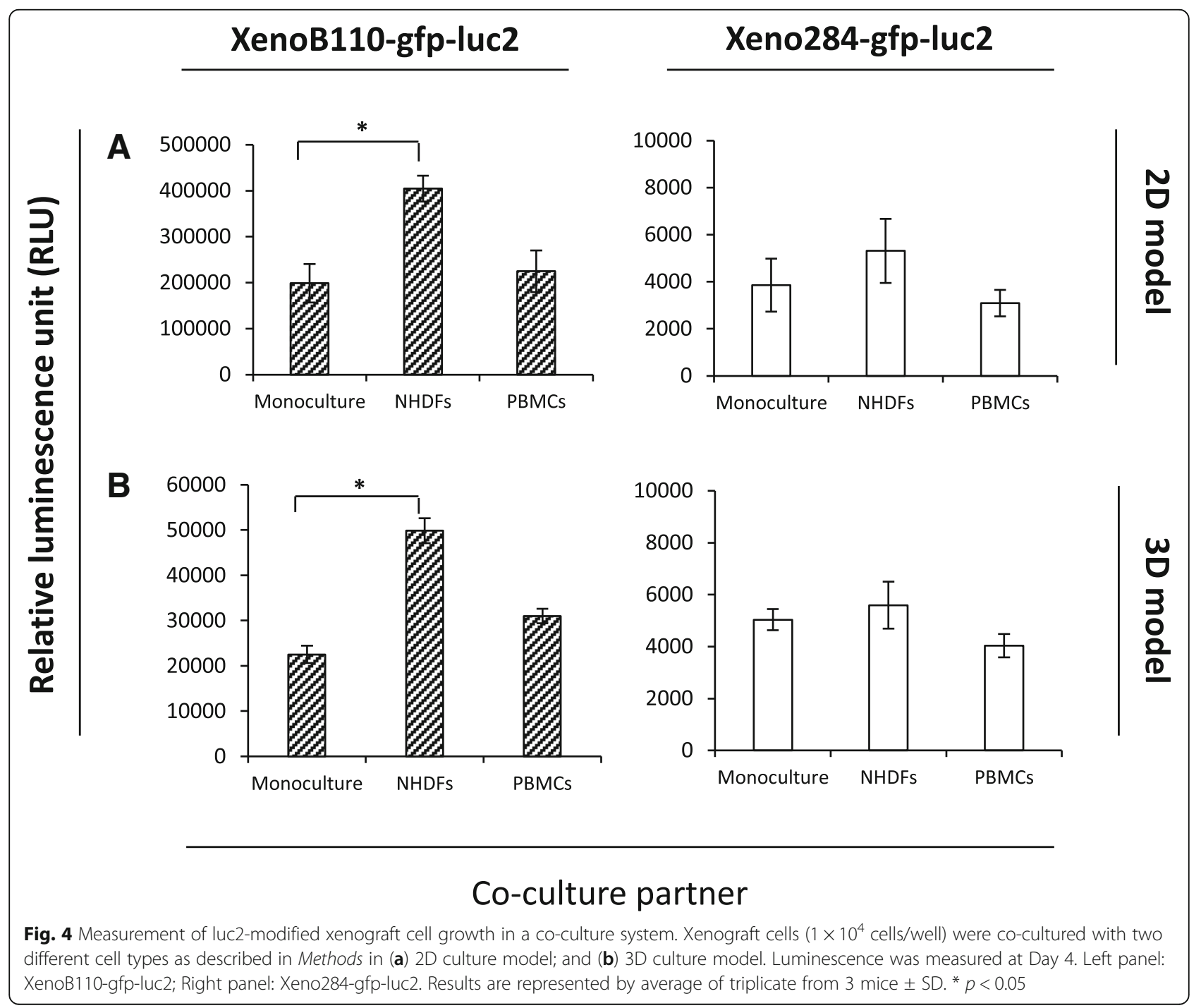




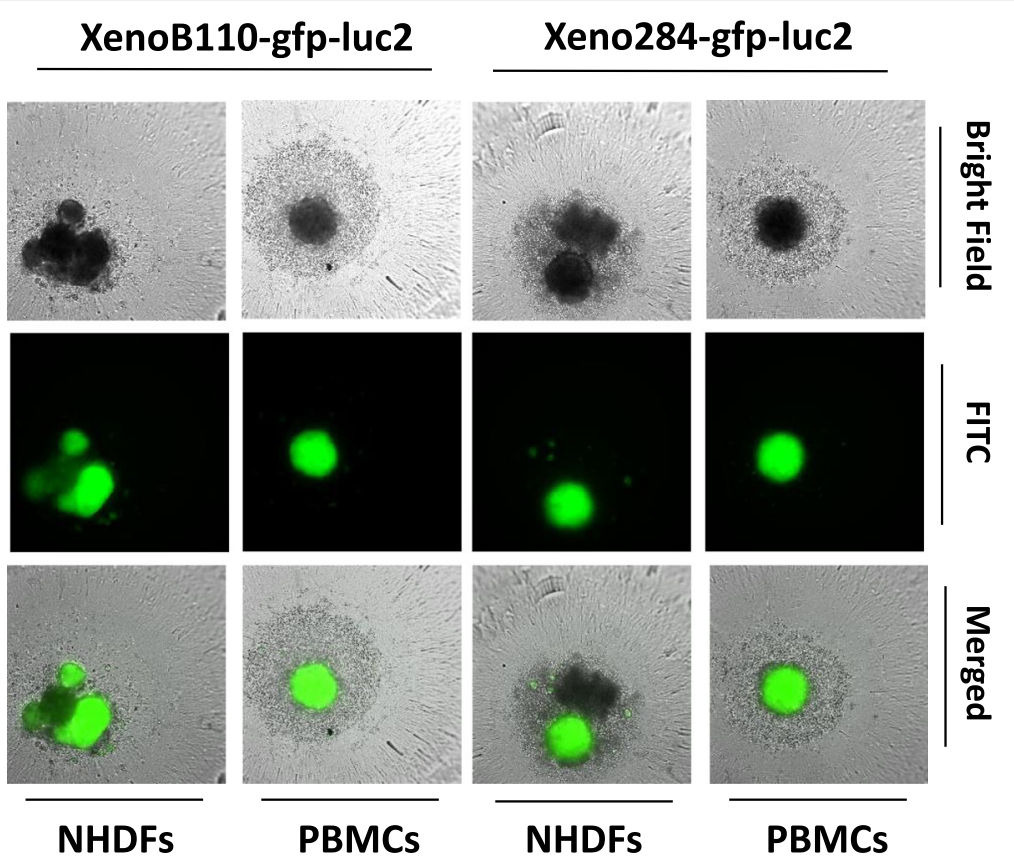

Fig. 5 Microscopic examination of xenograft cells co-cultured with NHDFs and PBMCs in 3D culture model. 3D spheroids from xenograft cellNHDF co-culture tended to form irregular shapes unlike those of xenograft cell-PBMC co-culture which were single, smooth and uniformed aggregates. (left panel: XenoB110-gfp-luc2; right panel: Xeno284-gfp-luc2)

establishing stable cell lines of NPC and allowed the use of xenografts which could not be propagated indefinitely as cell lines to be used for in vitro assays. This assay could be performed without lysing the cells, thereby allowing repeated and multiparametric measurements at multiple time-points in a single well (Fig. 1c). This assay could also capture the different growth patterns of culture models as shown in Fig. 1c. Both B110 and 284, the 3D culture models stopped growing at day 4, possibly due to the nutrient deprivation as basal RPMI1640 medium without any supplementation was used for culturing 3D spheroids. The luminescent signal was found to be stable up to $60 \mathrm{~min}$, and D-Luciferin at working concentration was not toxic to the cells after 24-h exposure (Fig. 2c and d). We also showed that the assay is robust and could be applied on different cell model systems. Furthermore, it was reliably used for multiple applications, including drug/ inhibitor screening and growth measurement in a co-culture system (Figs. 3 and 4).

The luciferase/luciferin system has been adopted in various cell-based assays, and is widely used in biomedical research. This assay is sensitive, non-toxic, suitable for various applications and does not interfere with downstream applications [10, 13]. Conventional luciferasebased assays use exogenous or endogenous luciferase to generate bioluminescence upon the addition of an external substrate (luciferin). The amount of emitted light depends on the concentration of ATP, a widely accepted marker of viable cells that is also the limiting reactant in luciferase-based assays. Non-viable cells are unable to synthesize ATP and rapidly exhaust the existing ATPs through their endogenous ATPases. Hence, the amount of cellular ATP in viable cells determines the intensity of luminescent signal which is proportionate to the viable cell number $[14,15]$.

In addition to luminescence, GFP fluorescence of the transduced cells could also be detected, in contrast to the commercially available luminescence-based assays (Fig. 5 and Additional file 2: Figure S2C). The current assay utilizes dual $g f p$ and $l u c 2$ reporters to provide more cellular information. The endogenous luciferase (encoded by $l u c 2$ reporter gene) in viable cells reacts chemically with an addition of luciferin to generate a luminescent signal, whereas the GFP signal (encoded by $g f p$ reporter gene) provides a fluorescent visualization of transduced tumour cells. The 2-in-1 detection in XenoLuc assay helps researchers to differentiate cell types in a co-culture system. GFP signal intensity could also be determined using imaging software as a second measure of cell proliferation. It is noteworthy that traditional metabolic-based viability assays such as MTT, MTS and XTT do not discriminate the metabolic activity between cancer and stromal cells when they are cultured together. This results in an inability to measure accurately the viability of either cell population when the above metabolic assays are used in co-culture systems [16]. As luciferase and GFP expressions are confined within transduced cancer cells, measurement of luminescence 
and/or GFP fluorescence will accurately reflect cell proliferation changes in co-cultures. However, it should be noted that GFP fluorescence could not effectively measure the growth of 3D spheroid culture (Additional file 2: Figure S2C) unlike luminescence (Fig. 1c). This suggests that GFP fluorescence is less sensitive, possibly because the GFP signals do not penetrate well enough through the cells from within the 3D spheroids.

To demonstrate that XenoLuc assay is comparable, if not better than commercially available luminescent assays, we performed some of the experiments using our assay in parallel with CellTiter-Glo and RealTime-Glo kits from Promega, USA. Of note, the rate-limiting factor of both commercial assays is cellular ATP, while the supplied luciferin and luciferase are in excess. On the other hand, the limiting factor in XenoLuc could be the endogenous luciferase, cellular ATP, or both (Fig. 2a). By using these commercial kits as a benchmark, we showed that XenoLuc assay met several important criteria as a cell proliferation or viability assay, mainly being highly sensitive, rapid, non-toxic, quantitative, and yields stable signals (Fig. 2b-d). In addition, a comparison of the features among these assays was tabulated in Table 1. Despite having lower signal intensity, XenoLuc assay has several notable advantages over other luminescent assays which include having a lower cost per reaction but with its performance and robustness comparable to that of commercial assays. Thus, the XenoLuc assay appears to be the more cost-effective option for researchers. More importantly, XenoLuc assay may be used to measure specifically the proliferation of $g f p$ and luc2-labeled cells of interest in multi-cellular systems such as co-cultures, unlike existing luminescent assays.

As a proof that XenoLuc assay could be a reliable tool for high-throughput screening for drug discovery, we tested the effect of cisplatin on the viability of our NPC xenograft cells. XenoLuc assay was able to show that although both transduced xenograft cells were sensitive to cisplatin, Xeno284-gfp-luc2 cells displayed some resistance to it as consistently observed in both $2 \mathrm{D}$ and $3 \mathrm{D}$ cultures. This observation could be attributed to the difference in disease type: Xeno-284 was established from recurrent NPC while XenoB110 was from untreated primary NPC. In the former, we speculate that the patient might have developed cisplatin resistance as a result of repetitive chemotherapy.

In the past decades, tumour cell monoculture has been used as a model for drug screening and the study of cancer biology. As increasing attention is drawn towards the complexity of tumour microenvironment, a co-culture model is believed to be more physiologically relevant for cancer research [2, 3]. The growth of XenoB110-gfpluc2 cells was markedly enhanced when co-cultured with NHDFs (Fig. 4). In addition to luminescence data, it was also validated by the GFP fluorescence intensity analysis that the NHDFs promoted the growth of XenoB110-gfpluc2 cells (Additional file 4: Figure S4). Unlike luminescence measurement that is in direct proportion to ATP levels [17], the fluorescence intensity analysis is not affected by the cellular ATP levels from the co-cultures. This growth enhancement was likely resulted from the activation of proliferative pathways upon direct cell-cell contact. NHDFs have been used as feeder cells to support cancer cell growth and to simulate epithelialstromal cell interaction and paracrine signalling in an in vitro culture setting [18-21]. In addition, we also used PBMCs as the co-culture partner for xenograft cells. There were varying results in the growth of NPC xenograft cells when cultured in 2D and 3D models (Fig. 4). It could be due to the heterogeneity within PBMCs population as well as differential cell-cell contacts due to culture techniques.

\section{Conclusions}

In conclusion, we have developed a specific, sensitive, real-time, non-toxic, and cost-effective luminescent assay to measure the proliferation of luc2-transduced cells. This robust assay known as XenoLuc assay could be used for multiple applications ranging from a simple experiment such as cell doubling time measurement, to complicated studies such as cell activation and proliferation or drug sensitivity/resistance screening in a coculture system. This assay may serve as an important tool to specifically examine the role of a selected cell types in complex co-culture systems as well as for drug screening experiments.

Table 1 Comparison of XenoLuc assay with commercial luminescent assays

\begin{tabular}{|c|c|c|c|c|c|c|}
\hline Assay & Lytic/ Non-lytic & Price per reaction $^{a}$ & Incubation time & Signal intensity & Signal stability & Linearity $\left(R^{2}\right)$ \\
\hline XenoLuc (In-house) & Lytic & RM 0.013 (USD 0.003) & $15 \min$ & 36,133 & $>1 \mathrm{~h}$ & 0.9915 \\
\hline CellTiter-Glo (Promega, USA) & Lytic & RM 0.20 (USD 0.05) & $1 \mathrm{~h} 15 \mathrm{~min}$ & 165,000 & $>1 \mathrm{~h}$ & 0.9963 \\
\hline XenoLuc (In-house) & Non-lytic & RM 0.013 (USD 0.003) & $10 \mathrm{~min}$ & 120,960 & $\geq 1 \mathrm{~h}$ & 0.999 \\
\hline RealTime-Glo (Promega, USA) & Non-lytic & RM 1.86 (USD 0.46) & $1 \mathrm{~h} 15 \mathrm{~min}$ & 279,400 & $\geq 1 \mathrm{~h}$ & 0.9835 \\
\hline
\end{tabular}

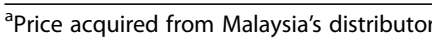




\section{Methods}

\section{Plasmid constructs and reagents}

Lentiviral constructs (pMDL, RSV-REV, and VSVg) and gfp-luc2 DNA transfer plasmid were kindly provided by Marco Herold (Walter and Eliza Hall Institute of Medical Research, Melbourne, Australia). RPMI 1640 (\#31800-022), fetal bovine serum (FBS) (\#10082-139), GlutaMAX supplement (\#35050-061), 0.05\% Trypsin-EDTA (\#25300-120), Pen-Strep (\#15140-122), Pen-Strep-Fungizone (\#15240062), B-27 supplement (\#17504-001), Insulin-TransferrinSelenium (ITS) (\#41400-045), Fibroblast growth factor (FGF)-Basic (bFGF) (\#PHG0261), and Epidermal growth factor (EGF) (\#PHG0311) were obtained from Gibco, USA. Hydrocortisone (\#H0135), Collagenase Type II (\#C6885), DPBS (\#D5652), HEPES (\#H3375), and Sodium Bicarbonate (\#S5761) were purchased from Sigma-Aldrich, USA. DNase I (\#90083) and Lipofectamine 2000 (\#11668-027) were purchased from Thermo Fisher Scientific, USA. Collagenase/Dispase (\#11097113001) was obtained from Roche, USA. Rho kinase (ROCK) inhibitor (\#SCM075) and Polybrene (\#TR-1003-G) were obtained from Merck Millipore, USA. XenoLight D-Luciferin substrate (\#122799) was purchased from PerkinElmer, USA and stored in small aliquots at $-20{ }^{\circ} \mathrm{C}$ in the dark. CellTiter 96 AQueous One Solution Cell Proliferation Assay (MTS) (\#G3580), CellTiter-Glo Luminescent Cell Viability Assay (\#G7571), and RealTimeGlo MT Cell Viability Assay (\#G9711) were from Promega, USA. Cisplatin (\#15663-27-1) was purchased from Acros Organics, USA. RBC lysis solution was purchased from Qiagen, USA. All reagents were dissolved, stored, and used according to the manufacturer's instruction.

\section{Cells and culture conditions}

Primary normal human dermal fibroblasts (NHDFs) (\#C-12300) were obtained from PromoCell, Germany and maintained using the medium and supplements supplied in the fibroblast growth medium kit (\#C-23110) (PromoCell, Germany). HEK293T cells (\#CRL-3216, ATCC, USA) were maintained in high glucose DMEM supplemented with 10\% FBS (DMEM-10). Human peripheral blood mononuclear cells (PBMCs) were isolated from the blood samples collected from three donors via Ficoll-Paque (GE Healthcare, USA) centrifugation. All donors had agreed and signed the consent forms.

\section{Tissue dissociation and xenograft cell culture}

Human NPC xenografts (XenoB110 and Xeno284) from NSG mice were processed as previously described by our group $[8,22]$. All experiments were performed in accordance with the protocols approved by Animal Care and Use Committee (ACUC), Ministry of Health, Malaysia. Briefly, mice were humanely euthanized using carbon dioxide gas (flow rate of between 1.3 to $3.8 \mathrm{~L} /$ min) or cervical dislocation by trained personnel using protocols approved by the Animal Care and Use Committee. Harvested xenograft tumours were transferred to cold DPBS supplemented with 1X Pen-Strep-Fungizone, and processed immediately. Surrounding blood capillaries, fat, and/or necrotic tissue were removed and washed twice with DPBS with $1 \mathrm{X}$ Pen-Strep-Fungizone. The tumours were minced into 2 to 4-mm fragments and incubated with the appropriate dissociation solution $(2 \mathrm{mg} /$ $\mathrm{mL}$ Collagenase Type II and $200 \mathrm{U} / \mathrm{mL}$ DNase I for XenoB110; $1 \mathrm{mg} / \mathrm{mL}$ Collagenase/Dispase for Xeno284) on a belly dancer shaker with a constant agitation for 1$2 \mathrm{~h}$ at $37^{\circ} \mathrm{C}, 5 \% \mathrm{CO}_{2}$. This was followed with an addition of complete growth media (RPMI-10, 1X Pen-StrepFungizone, 1X B-27 Supplement, 1X ITS, $10 \mu \mathrm{M}$ ROCK Inhibitor, $10 \mathrm{ng} / \mathrm{mL} \mathrm{EGF}$, and $10 \mathrm{ng} / \mathrm{mL} \mathrm{bFGF}$ ), after which the entire suspension was filtered through $40 \mu \mathrm{m}$ nylon mesh cell strainer (BD Falcon, USA). The released cells were centrifuged at $800 \mathrm{rpm}$ for $5 \mathrm{~min}$. RBC lysis solution was added onto the cells prior to centrifugation at $800 \mathrm{rpm}$ for $5 \mathrm{~min}$. The cells were then processed with the mouse cell depletion kit (MACS Miltenyi Biotec \#130-104-694) following the manufacturer's instruction to enrich for human NPC xenograft cells. The enriched cells were cultured in the above mentioned complete growth medium for 2D monolayer culture, whereas basal RPMI-10 was used for 3D spheroid culture. The cells were detached using $0.05 \%$ Trypsin-EDTA at 70-80\% confluence and were sub-cultured at 1:3 dilutions. To our best experience, xenograft cells could be maintained up to passage four (P4) in vitro in monolayer culture, and spheroids could be formed by medium exchange and using a commercial spheroid plate. To minimize biological variations, we only used freshly isolated xenograft cells which were passaged for up to 2 times for experiments. We used the terms xenograft cells for these cells in contrast to xenograft tumours in mice.

\section{Lentivirus production and cell transduction}

$1 \times 10^{6}$ HEK293T cells were seeded on $10-\mathrm{cm}$ culture dishes and incubated for $24 \mathrm{~h}$. The gfp-luc2 lentiviral transfer vector with packaging and envelope plasmids (pMDL, RSV-REV and VSVg) were combined at a ratio of $4: 2: 1: 1$, respectively, and mixed with Lipofectamine 2000 for transfection according to the manufacturer's protocol. The viral supernatant was collected at 24, 48 and $72 \mathrm{~h}$ post-transfection. Cellular debris was pelleted by centrifugation at $1500 \mathrm{rpm}$ for $5 \mathrm{~min}$ at $4{ }^{\circ} \mathrm{C}$ followed by filtration using PVDF Millex-HV filter, $0.45 \mu \mathrm{m}$ (Millipore \#SLHV033RS). The filtered lentivirus supernatant was then concentrated using Lenti-X Concentrator (Clontech \#PT4421-2) according to the manufacturer's instruction. For cell transduction, the mouse cell-depleted xenograft cells were seeded on a $10-\mathrm{cm}$ culture dish and transduced with the concentrated lentivirus at MOI 2.0 in 
the presence of $10 \mu \mathrm{g} / \mathrm{mL}$ Polybrene for $24 \mathrm{~h}$. GFP positive cells were selected using a FACSAria SORP (BD Biosciences, USA).

\section{XenoLuc assay}

XenoLuc assay was performed as follows for both 2D and 3D culture experiments. At each time point, 2X DLuciferin substrate diluted in RPMI-10 was dispensed into each cell-containing well at 1:1 ratio. The plate was gently agitated and incubated at room temperature in the dark for $10 \mathrm{~min}$ to stabilize the luminescence. The luminescent signal of each plate was then read by EnVision multi-label plate reader (PerkinElmer) using the ultrasensitive mode. Following the completion of a time-point reading, the DLuciferin containing medium was removed from the well. The cells were washed two times gently with $200 \mu \mathrm{L}$ RPMI-10, and then replenished with $100 \mu \mathrm{L}$ of fresh complete media until the subsequent reading. Alternatively, images of GFP-expressing xenograft cells from each well were captured using IN Cell Analyzer 2000, and the GFP fluorescence intensity was measured and compared using the IN Cell Developer software. For the lytic-based format of XenoLuc assay, the protocol described by Oba and co-workers was modified and adapted [23]. Briefly, PBS supplemented with $1 \%$ Triton X-100 and $1 \mathrm{X}$ protease inhibitor cocktail (Millipore \#539134) was used as cell lysis buffer and the lysis was performed for $10 \mathrm{~min}$. After lysis, the assay buffer containing $5 \mathrm{mM} \mathrm{MgCl}_{2}, 100 \mathrm{mM}$ Tris- $\mathrm{HCl}(\mathrm{pH} 7.8)$ and $2 \mathrm{X}$ D-Luciferin substrate was added to the well to generate the luminescence. The plate was immediately measured after 15-min incubation at room temperature in the dark.

\section{CellTiter-Glo and RealTime-Glo}

CellTiter-Glo (end-point assay) and RealTime-Glo (continuous assay) were performed according to the manufacturer's instructions. To examine if the XenoLight DLuciferin substrate reagent was toxic to the xenograft cells, cell viability assay (CellTiter 96 Aqueous One) was performed on xenograft cells which had been exposed to the substrate for $24 \mathrm{~h}$.

\section{Applications of XenoLuc assay}

We evaluated the suitability of the assay for inhibitor/drug screening and co-culture studies using both 2D and 3D culture models. For the drug screening study, cisplatin was used as the standard drug. Xenograft cells were similarly plated in 2D and 3D culture conditions as described above. Cells were then treated with various concentrations of cisplatin $(0,0.78,1.56,3.13,6.25,12.5,25$, and $50 \mu \mathrm{M})$ for $72 \mathrm{~h}$ followed by the XenoLuc assay. Prior to coculturing, both NHDFs and PBMCs were gammairradiated separately at $35 \mathrm{~Gy}$. For both $2 \mathrm{D}$ and $3 \mathrm{D}$ cultures, 10,000 xenograft cells were seeded simultaneously with the irradiated co-culture partner cells at 1:1 ratio into the same well. After an incubation of 4 days, XenoLuc assay was performed to measure the proliferation of xenograft cells in the presence of either irradiated NHDFs or PBMCs.

\section{Statistical analysis}

For each experiment using xenograft cells, three tumours were harvested from three separate mice, and each resulting xenograft cells were seeded into three wells per isolate of xenograft cells for subsequent experiments. Each data point was represented by average of triplicate from 3 mice \pm SD. GraphPad Prism 6 software (GraphPad Software Inc.) was used to calculate the $\mathrm{IC}_{50}$ value of cisplatin against xenograft cells. $R^{2}$ value was also determined to show the linear association between two parameters in a linear curve. SPSS version 22.0 software (IBM SPSS, USA) was used for analysis of statistical significance. One-way analysis of variance with Bonferroni correction and Student's $t$ test were used to determine if the differences between two different groups are significant. The results presented are mean \pm standard deviations. $p<0.05$ was considered statistically significant.

\section{Additional files}

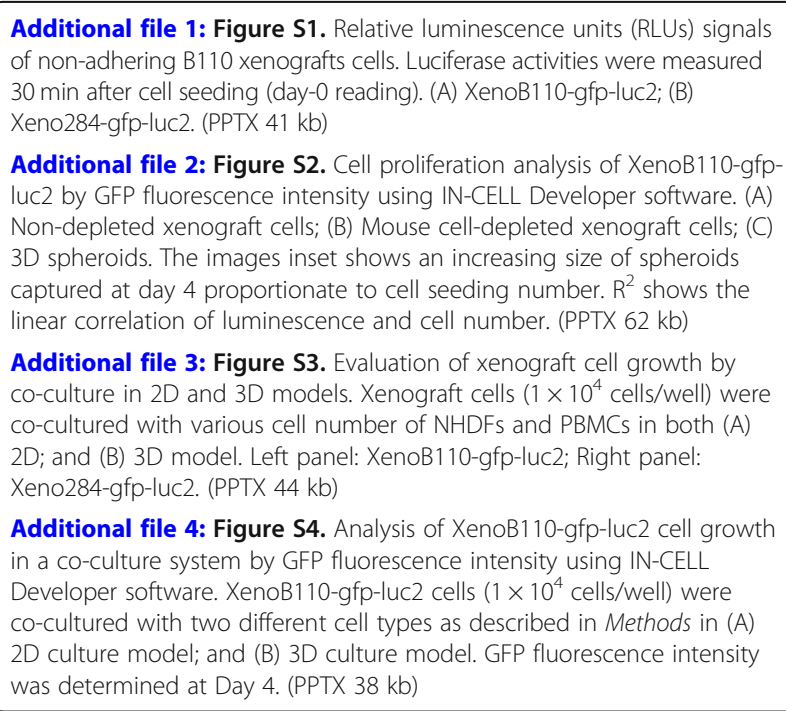

\section{Abbreviations}

2D: Two-dimensional; 3D: Three-dimensional; ATP: adenosine triphosphate; EBER: Epstein-Barr virus-encoded small RNA; EBV: Epstein-Barr virus;

ECM: extracellular matrix; EGF: epidermal growth factor; FACS: fluorescenceactivated cell sorting; FGF: fibroblast growth factor; GFP: green fluorescence protein; H\&E: Haematoxylin and Eosin; ITS: Insulin-Transferrin-Selenium; LCA: Leukocyte common antigen; luc: luciferase; NHDFs: normal human dermal fibroblasts; NPC: nasopharyngeal carcinoma; NSG: NOD scid gamma; PAN-CK: Cytokeratin Pan; PBMC: peripheral blood mononuclear cell; PBS: phosphate buffered saline; PDTX: patient-derived tumour xenograft; $\mathrm{RBC}$ : red blood cells; RFU: relative fluorescence unit; RLU: relative luminescence unit; ROCK: Rho kinase 


\section{Acknowledgements}

The authors would like to thank the Director General of Health Malaysia for his permission to publish this study and the Director of the Institute for Medical Research for her support. We would like to acknowledge Specific Pathogen Free facility, Biobank and Molecular Pathology Unit, IMR staff for their technical assistance and support in this project as well as Dr. Susan LL Hoe for her critical appraisal of the manuscript.

\section{Authors' contributions}

SYT, KL, MFCM and CTL performed the experiments. SYT, KL, MFCM, NAA and MM analysed the data. SYT and KL drafted the manuscript. SYT, MA, and ASBK conceived the study, participated in its design and reviewed the manuscript. All authors read and approved the final manuscript.

\section{Funding}

This work was funded by the Ministry of Health Malaysia. The funder did not have any role in the design of the study, data collection, analysis, and interpretation of data or in writing the manuscript.

\section{Availability of data and materials}

The datasets used and/or analysed during the current study are available from the corresponding author on reasonable request.

\section{Ethics approval}

All procedures and experimental protocols involving the use of NPC PDXs in this study were in accordance with the ethical standards of and approved by the Animal Care and Use Committee (ACUC), Ministry of Health, Malaysia [(ACUC/KKM/02 (03/2013), (05/2016), (16/2016) and (03/2017)]. The use of human blood specimens in this study was approved by the Medical Research and Ethics Committee (MREC), Ministry of Health, Malaysia [reference number: KKM/NIHSEC/P18-1062(10)]. Informed consent was obtained from all participating human subjects.

\section{Consent for publication}

Not applicable.

\section{Competing interests}

SYT, MFCM, MM, NAA, TLC, MA, ASBK are co-inventors of a patent filed related to the contents of this manuscript. (Khoo ASB, Norazlin AA, Teow SY, Mohd Firdaus CM, Marini M, Chu TL, Munirah A. Isolated nasopharyngeal carcinoma cells and derivatives prepared thereof. Malaysian Patent PI 2018702497 filed July 17, 2018 (Patent Pending).

\section{Author details}

'Molecular Pathology Unit, Cancer Research Centre, Institute for Medical Research, National Institutes of Health (NIH Complex), Ministry of Health Malaysia, Level 4, Block C7, No: 1, Jalan Setia Murni U13/52, Section U13, Setia Alam, 40170 Shah Alam, 50588 Kuala Lumpur, Selangor, Malaysia. ${ }^{2}$ Present Address: Department of Medical Sciences, School of Healthcare and Medical Sciences, Sunway University, 47500 Bandar Sunway, Selangor Darul Ehsan, Malaysia.

Received: 4 October 2018 Accepted: 20 May 2019

Published online: 14 June 2019

\section{References}

1. Zang R, Li D, Tang IC, Wang J, Yang ST. Cell-based assays in highthroughput screening for drug discovery. Int J Biotechnol Wellness Ind. 2012;1:31-51.

2. Lovitt C, Shelper T, Avery V. Advanced cell culture techniques for cancer drug discovery. Biology. 2014;3(2):345-67.

3. Weigelt B, Ghajar C, Bissell M. The need for complex 3D culture models to unravel novel pathways and identify accurate biomarkers in breast cancer. Adv Drug Deliv Rev. 2014;69-70:42-51.

4. Zhang Y, Schedle A, Matejk M, Rausch-Fan X, Andrukhov O. The proliferation and differentiation of osteoblasts in co-culture with human umbilical vein endothelial cells: an improved analysis using fluorescenceactivated cell sorting. Cell Mol Biol Lett. 2010;15(4):517-29.

5. Goers L, Freemont P, Polizzi K. Co-culture systems and technologies: taking synthetic biology to the next level. J R Soc Interface. 2014;11(96):20140065.
6. Edmondson R, Broglie J, Adcock A, Yang L. Three-dimensional cell culture systems and their applications in drug discovery and cell-based biosensors. Assay Drug Dev Technol. 2014;12(4):207-18.

7. Breslin S, O'Driscoll L. Three-dimensional cell culture: the missing link in drug discovery. Drug Discov Today. 2013;18(5-6):240-9.

8. Hoe SLL, Tan LP, Aziz NA, Liew K, Teow SY, Razak FRA, et al. CD24, CD44 and Epcam enrich for tumour-initiating cells in a newly established patientderived xenograft of nasopharyngeal carcinoma. Sci Rep. 2017;7(1):12372.

9. Leitão JM, Esteves da Silva JC. Firefly luciferase inhibition. J Photochem Photobiol B. 2010;101(1):1-8.

10. Duellman SJ, Zhou W, Meisenheimer P, Vidugiris G, Cali JJ, Gautam P, et al. Bioluminescent, nonlytic, real-time cell viability assay and use in inhibitor screening. Assay Drug Dev Technol. 2015;13(8):456-65.

11. Fraga $H$. Firefly luminescence: a historical perspective and recent developments. Photochem Photobiol Sci. 2008:7(2):146-58.

12. Tiffen JC, Bailey CG, Ng C, Rasko JE, Holst J. Luciferase expression and bioluminescence does not affect tumor cell growth in vitro or in vivo. Mol Cancer. 2010;9:299.

13. Adams ST, Miller SC. Beyond D-luciferin: expanding the scope of bioluminescence imaging in vivo. Curr Opin Chem Biol. 2014;0:112-20.

14. Riss TL, Moravec RA, Niles AL, Duellman S, Benink HA, Worzella TJ, et al. Cell viability assays. 2013 may 1 [updated 2016 Jul 1]. In: Sittampalam GS, Coussens NP, Nelson $\mathrm{H}$, et al., editors. Assay guidance manual. Bethesda: Eli Lilly \& Company and the National Center for advancing translational Sciences; 2004.

15. Niles A, Moravec R, Riss T. In vitro viability and cytotoxicity testing and same-well multi-parametric combinations for high throughput screening. Curr Chem Genomics. 2009;3:33-41.

16. Yang $N$, Yan $T$, Zhu $H$, Liang $X$, Leiss L, Sakariassen $P$, et al. A co-culture model with brain tumor-specific bioluminescence demonstrates astrocyteinduced drug resistance in glioblastoma. J Transl Med. 2014;12:278.

17. Posimo JM, Unnithan AS, Gleixner AM, et al. Viability assays for cells in culture. J Vis Exp. 2014:83:e50645.

18. Friboulet L, Pioche-Durieu C, Rodriguez S, et al. Recurrent overexpression of c-IAP2 in EBV-associated nasopharyngeal carcinomas: critical role in resistance to toll-like receptor 3-mediated apoptosis. Neoplasia. 2008;10(11): 1183-94.

19. Vérillaud B, Gressette M, Morel Y, et al. Toll-like receptor 3 in Epstein-Barr virus-associated nasopharyngeal carcinomas: consistent expression and cytotoxic effects of its synthetic ligand poly(a:U) combined to a Smacmimetic. Infect Agents Cancer. 2012;7:36.

20. Fang X, Sittadjody S, Gyabaah K, Opara E, Balaji K. Novel 3D co-culture model for epithelial-stromal cells interaction in prostate cancer. PLoS One. 2013:8(9):e75187.

21. Kayamori K, Katsube K, Sakamoto K, Ohyama Y, Hirai H, Yukimori A, et al. $\mathrm{NOTCH} 3$ is induced in cancer-associated fibroblasts and promotes angiogenesis in oral squamous cell carcinoma. PLoS One. 2016;11(4): e0154112.

22. Hoe SLL, Tan LP, Jamal J, Peh SC, Ng CC, Zhang WC, et al. Evaluation of stem-like side population cells in a recurrent nasopharyngeal carcinoma cell line. Cancer Cell Int. 2014;14:101.

23. Oba Y, Ojika M, Inouye S. Firefly luciferase is a bifunctional enzyme: ATPdependent monooxygenase and a long chain fatty acyl-CoA synthetase. FEBS Lett. 2003;540(1):251-4.

\section{Publisher's Note}

Springer Nature remains neutral with regard to jurisdictional claims in published maps and institutional affiliations. 